\title{
Tie1, The Orphan Receptor No Longer in the Shadows
}

\section{Nikolett M. Biel, PhD}

Department of Pathology, University of Florida College of Medicine, Gainesville, FL 32610, USA

Email:nmolnar@ufl.edu

\begin{abstract}
Angiogenesis, the formation of new blood vessels from pre-existing ones, occurs during physiological conditions such as wound healing, pregnancy and the menstrual cycle. It is also a fundamental process in pathological conditions such as tumor development, ocular disease, diabetes, atherosclerosis and arthritis. Angiogenesis as a therapeutic target (especially Vascular Endothelial Growth Factor (VEGF) inhibitors) has been heavily pursued in numerous cancer settings with promising results yet fallen short of its expectations. The discovery of the endothelial cell specific Angiopoietin/Tie axis and its role in normal and pathological angiogenesis led to the development of second-generation anti-angiogenic agents. Most of the effort has been to target the pro-angiogenic factor Ang-2 and its receptor Tie2. The present research highlight focuses on a study by D'Amico et al., "Tie1 deletion inhibits tumor growth and improves angiopoietin antagonist therapy" published in the Journal of Clinical Investigation, that demonstrates for the first time the therapeutic benefit of targeting the orphan receptor, Tie1, involved in the complex biological axis of the Angiopoietin/Tie2 system in angiogenesis. This study shows tremendous implications for future considerations in anti-angiogenic therapy for cancer and other angiogenesis related diseases.
\end{abstract}

Keywords: Angiogenesis, Angiopoietin, Anti-angiogenic therapy, Tie1

\section{Introduction}

Normal blood vasculature, arteries and veins, are composed of endothelial and smooth muscle cells/or pericytes, collectively called periendothelial cells. In the adult, normal vasculature rarely proliferate, however upon stimulation involved with wound healing, pregnancy and the menstrual cycle the vasculature becomes quite active. In pathological conditions, such as cancer, the diseased tissue environment manipulates the vasculature. This causes high proliferation and remodeling, loose endothelial and smooth muscle cell (or pericyte) contacts therefore making the "diseased" vasculature distinctively different from normal vasculature.

A fundamental characteristic to all solid tumors is the need for sustain angiogenesis in order to survive and grow ${ }^{1}$. For this reason, targeting new blood vessel growth has been most prominent in cancer settings. Since 2004, several antiangiogenic agents targeting a key axis in angiogenesis, Vascular Endothelial Growth Factor (VEGF) and VEGF-Receptor, have been approved by the FDA for numerous cancer types. Antiangiogenic agents provide targeted and less toxic therapy for patients compared to the traditional cytotoxic chemo- and radiation therapies but not without their own side effects or lack of efficacy in some cases $^{2}$. While the field has made tremendous progress in the last 40 years, the complexity of pathological angiogenesis presents new obstacles.

The discovery of a new endothelial cell specific angiopoietin/Tie axis merits a new class of antiangiogenic agents in hopes to circumvent resistance to - and boost the efficacy of - VEGF targeted anti-angiogenic therapies used in the clinic. Angiopoietins, in humans, comprise of three ligands, Ang-1, -2 , and -4 and two receptors Tie1 and Tie2. Ang-1 and Ang-2 are the best studied of the three ligands; in general they both bind the same receptor, Tie2, on the same site with similar affinities and neither bind to Tie1. Ang-1 is considered the "keeper" of normal 
vasculature; its interaction with Tie2 leads to receptor phosphorylation and downstream signaling through Rho and $\mathrm{mDia}$ to inhibiting VEGF/VEGFR-2 mediated cell permeability via $\mathrm{src}^{3}$. The endothelium-specific vascular endothelial-phosphotyrosine phosphatase (VEPTP) has also been implicated in cross talk between the Ang-1/Tie2 and VEGF/VEGFR pathways ${ }^{4}$.

On the other hand, Ang-2 is thought of as a Tie2 agonist, however it has also been shown to act as an agonist, able to phosphorylate the receptor. The agonistic or antagonistic role of Ang-2 has been implicated by Tie1/Tie2 receptor interactions. In the lymphatic vessels, Tie1 is sparsely expressed therefore Tie2 heterodimers interact with the Ang-2 ligand causing agonistic effects ${ }^{5}$. However, in the blood vasculature, Tie1 is often expressed and Tie1/Tie2 heterodimers form upon which Ang-2 is incapable of Tie2 phosphorylation ${ }^{6}$. Ang-2 then serves as an antagonist and loosens cell-cell interactions between both endothelial-endothelial and endothelial-smooth muscle cells, thus destabilizing the vasculature. Ang- 2 is highly expressed in tumors therefore most of the focus has been on targeting the Ang-2/Tie2 axis in the last 10 years ${ }^{7}$.

\section{Results}

There is evidence in the literature that Tie1 plays a role in the differing actions of Ang-1 and Ang-2 and that it is an important factor in developmental angiogenesis especially lymphangiogenesis ${ }^{8-10}$. It has also been noted that expression of Tie1 is upregulated in the tumor microvasculature ${ }^{11}$. For the first time, D'Amico et al. in The Journal of Clinical Investigation show direct evidence for the therapeutic potential of Tie1 targeting as an anti-angiogenic approach ${ }^{12}$.

Deletion of either Angiopoietins or their receptors Tie1 and Tie2 result in embryonic lethality. Therefore, to study the role of Tie1 in postnatal angiogenesis, the authors created a conditional Tie1 knockout in the endothelial cells of mice. The authors elegantly demonstrate that the growth of several murine cancer cells lines, such as Lewis lung carcinoma (LLC), melanoma (B16) and lymphoma (EL4), are impaired in the Tie1 deleted mice by $\sim 50-75 \%$ compared to the control tumors. Additionally, the authors establish that along with tumor growth impairment, Tie1 deleted mice have a 30-50\% reduction in vascular area within the tumors, indicating that tumor cell induced angiogenesis is compromised.

Furthermore, Tie1 deficiency increased endothelial cell apoptosis in tumor blood vessels, supporting the role of Tie1 in endothelial cell survival. Concerned with normal blood vasculature, the author's compared vessels in the Tie1 wild type and deleted mice in several organs (ex. lungs, kidneys, heart, thyroid, small intestines) and did not find significant differences between the two groups. Furthermore, results confirmed normal kidney functions in both wild type and Tie1 deficient mice, suggesting that Tie1 is expendable in quiescent adult vasculature.

Interestingly, endothelial cell Tie1 deletion did not improve VEGF (mcr84) or VEGFR (DC101) directed antibody treatment of LLC, B16 or EL4 tumors even though there are examples of additive anti-angiogenic effects of Ang-2/Tie2 and VEGF/VEGFR targeted therapies ${ }^{13,14}$. The authors attribute this difference to the observation that Tie1 deficient mice have lower levels of the VEGF receptor, thus angiogenesis through VEGF-VEGFR is impaired compared to control mice. On the other hand, however, combination of Ang-2-Tie2 axis targeting (soluble Tie2 receptor) with the Tie1 deleted mice resulted in improved tumor growth inhibition than either strategy alone in the LLC tumor model.

Finally, anti-angiogenic agents had success not only in the cancer setting but also in angiogenesis dependent eye disease. In the last set of experiments the authors focused on a retinal angiogenesis model and demonstrated that Tie1 deletion impairs postnatal retinal angiogenesis. Furthermore, similar results to the tumors 
angiogenesis were seen when Ang-2-Tie2 axis with Tie1 deletion was combined in the retinal

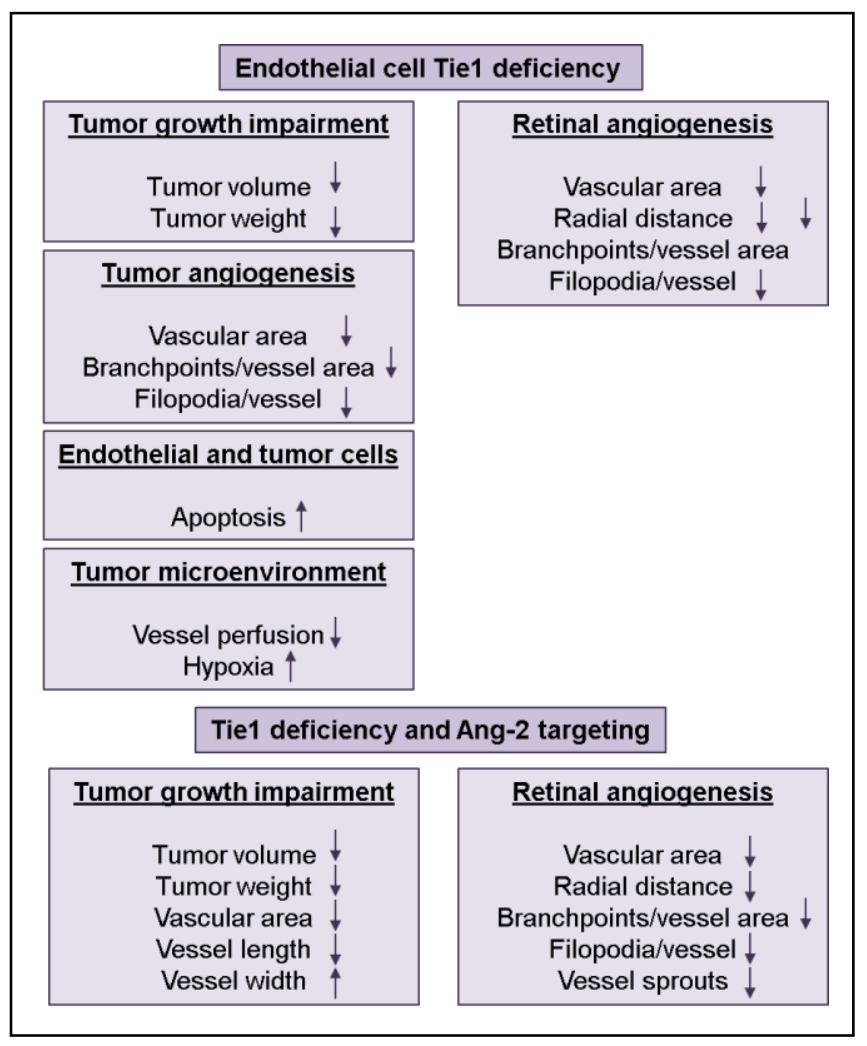

\section{References}

1. Folkman J. Angiogenesis: an organizing principle for drug discovery? Nat Rev Drug Discov 2007; 6:273-86.

2. Bergers G. and Hanahan D. Modes of resistance to anti-angiogenic therapy. Nat Rev 2008; 8:592-603.

3. Gavard, J., V. Patel, and J.S. Gutkind, Angiopoietin-1 prevents VEGF-induced endothelial permeability by sequestering Src through mDia. Dev Cell, 2008; 14:2536.

4. Hayashi, M., et al., VE-PTP regulates VEGFR2 activity in stalk cells to establish endothelial cell polarity and lumen formation. Nat Commun, 2013; 4: 1672.

5. Song, S.H., et al., Tie1 regulates the Tie2 agonistic role of angiopoietin-2 in human angiogenesis model, leading to $\sim 60 \%$ reduction in vascular area.

Figure 1. Summary of the Effects of Tie1 deficiency in the tumor and ocular disease settings.

\section{Concluding Remark}

The therapeutic implications of this study are significant for all angiogenesis related diseases; however it has significant potential in cancer therapeutics especially during an era of antiVEGF therapy resistance and thus efforts to develop new anti-angiogenic targets. Currently, there is only one agent targeting Tie1, a human monoclonal antibody DX-2240 (Sanofi-Aventis), in preclinical development; no therapeutic results have been reported on this agent yet.

\section{Acknowledgements}

I'd like to thank Dr. Stephan C. Jahn and the reviewers for their helpful comments in preparing this research highlight.

lymphatic endothelial cells. Biochem Biophys Res Commun, 2012; 419:281-6.

6. Seegar, T.C., et al., Tie1-Tie2 interactions mediate functional differences between angiopoietin ligands. Mol Cell, 2010; 37:643-55.

7. Molnar N. and Siemann DW. Angiopoietin-2 axis inhibitors: current status and future considerations for cancer therapy. Curr Angio 2013; 2:2-12.

8. Kim KL, Shin IS, Kim JM et al. Interaction between Tie receptors modulates angiogenic activity of angiopoietin-2 in endothelial progenitor cells. Cardiovas Res 2006; 72:394-402.

9. Seegar TC, Eller B, Tzvetkova-Robev D et al. Tie1-Tie2 interactions mediate functional differences between angiopoietin ligands. Mol Cell 2010; 37:643-55. 
10. Yu X, Seegar TC, Dalton AC et al. Structural basis for angiopoietin-1 mediated signaling initiation. Proc Natl Acad Sci USA 2013; 110:7205-10.

11. Harva $\mathrm{E}$ et al. Expression of endothelial cell specific receptor tyrosine kinases and growth factors in human brain tumors. Am J Pathol 1995; 146:368-378.

12. D'Amico $\mathrm{G}$ et al. Tie1 deletion inhibits tumor growth and improves angiopoietin antagonist therapy. J Clin Invest 2014; 124:824-834.

13. Hashizume $\mathrm{H}$ et al. Complementary actions of inhibitors of angipoietin-2 and VEGF on tumor angiogenesis and growth. Cancer Res 2010; 70:2213-2223.

14. Molnar $\mathrm{N}$ and Siemann DW. Combined Ang2 and VEGF Targeting Therapies in Renal Cell Carcinoma. J Cancer Ther 2013; 4: 1-6. 